\title{
ORIGINAL
}

\section{PREVALENCIA DE PARASITOSIS INTESTINALES EN LA COMUNIDAD RURAL APOSTADERO, MUNICIPIO SOTILLO, ESTADO MONAGAS, VENEZUELA.}

\section{PREVALENCE OF INTESTINAL PARASITOSES IN THE RURAL COMMUNITY APOSTADERO SOTILLO MUNICIPALITY, MONAGAS STATE, VENEZUELA.}

Brito Núñez Jesús David ${ }^{1 a}$. Landaeta Mejías Juan Antonio ${ }^{1 a}$. Chávez Contreras Andrea Neseva ${ }^{1 a}$. Gastiaburú Castillo Priscilla Katherine ${ }^{1 a}$. Blanco Martínez Ytalia Yanitza ${ }^{2 b}$.

\section{RESUMEN}

INTRODUCCIÓN: Las enfermedades parasitarias a nivel intestinal aún tienen un papel importante en salud pública y son uno de los problemas médicos importantes, constituyendo una de las principales causas de morbilidad en niños en Latinoamérica, así también Venezuela.

Objetivo: Determinar la prevalencia de parasitosis intestinales en niños menores de 15 años en la población estudiada.

METODOLOGÍA: El tipo de estudio fue observacional, descriptivo y de corte transversal, cada participante fue evaluado clínicamente y mediante un análisis coproparasitológico, las muestras fecales fueron estudiadas mediante examen directo en solución salina fisiológica y coloración temporal de lugol, y la aplicación de la Técnica de Kato. Dichas técnicas se realizaron directamente en comunidad rural.

RESULTADOS: Se evaluaron 64 niños entre 0-15 años de edad, de ellos: 51,6\% masculinos y 48,4\% femeninos. $87,5 \%$ presentaron manifestaciones clínicas, siendo el bruxismo más frecuente, seguido de dolor abdominal. El hallazgo de parasitosis intestinales general fue $92,20 \%$. Se diagnosticaron parasitosis por 14 especies diferentes, de ellos 9 eran protozoarios y 5 helmintos.

CONCLUSIÓN: Se determinó una alta prevalencia de parasitosis intestinales en niños menores de 15 años, los helmintos más frecuentes fueron: Ascaris lumbricoides, Trichuris trichiura y Ancilostomideos con 72,9\%, 47,5\% y $33,9 \%$ respectivamente. Entre los protozoarios y cromistas destacan: Blastocystis spp $(50,8 \%)$, Entamoeba coli $(28,8 \%)$ y Giardia lamblia $(23,7 \%)$. El poliparasitismo fue superior a $81,4 \%$ y las asociaciones más frecuentes fueron: Ascaris lumbricoides + Blastocystis spp (12,5\%), seguido por Entamoeba coli + Ascaris lumbricoides + Trichuris trichiura (6,3\%).

\section{ABSTRACT}

INTRODUCTION: The intestinal parasitic diseases still have an important role in terms of public health, and they are one of main medical problems, being one of the principle causes of morbidity in children in Latin America included Venezuela. Objectives: Establish the prevalence of intestinal parasites in children less than 15 years old in the studied population.

METHODOLOGY: The type of study was observational, descriptive and cross-sectional, each participant was evaluated clinically and through coproparasitologic test, fecal samples were studied by direct examination in physiological saline solution and temporary stained with lugol, and application of Kato-Katz's technique. These techniques were performed directly in the rural community.

RESULTS: 64 children between $0-15$ years old were evaluated; $51,6 \%$ male and $48,4 \%$ female. $87,5 \%$ had clinical manifestations, being bruxism the most frequent, followed by abdominal pain. The overall finding of intestinal parasites was $92,20 \%$. There were diagnosed parasitoses by 14 different species, 9 were protozoans and 5 were helminths.

CONCLUSION: It was determined a high prevalence of intestinal parasites in children under 15 years old, the most common helminths were: Ascaris lumbricoides, Trichuris trichiura and hookworm with $72,9 \%, 47,5 \%$ and $33,9 \%$ respectively. Among protozoans and chromists that stood out were Blastocystis spp (50,8\%), Entamoeba coli $(28,8 \%)$ and Giardia lamblia (23,7\%). Polyparasitism was higher than $81,4 \%$ and the most common associations were: Ascaris lumbricoides + Blastocystis spp. 12,5\%, followed by Entamoeba coli + Ascaris lumbricoides + Trichuris trichiura with $6,3 \%$.

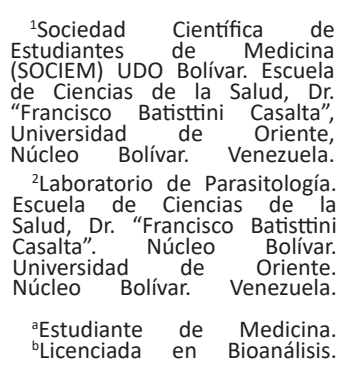

Correspondencia a:

Nombre: Brito Núñez Jesús David Correo electrónico:

jdbn_93@hotmail.com

Telf. $\bar{y}$ celular: 00584249721904

Palabras clave: Parasitosis $\begin{aligned} & \text { intestinales, } \\ & \text { comunidad }\end{aligned}$
rural. Keywords: Intestinal parasites, prevalence, rural community.

Procedencia y arbitraje: no comisionado, sometido a arbitraje externo.

Recibido para publicación: 15 de octubre 2016 Aceptado para publicación: 18 de noviembre 2017 Citar como: Rev Cient Cienc Med. 2017; 20(2): 7-14. 


\section{INTRODUCCIÓN}

Las enfermedades parasitarias a nivel intestinal continúan teniendo un papel importante en salud pública principalmente en países en vías de desarrollo. Los protozoos han resaltado como agentes con un gran potencial para generar epidemias desde hace unas décadas, por medio de agua y alimentos ${ }^{1}$, aunado a otras causas como viviendas precarias sin instalaciones sanitarias adecuadas, alto nivel de hacinamiento, bajo nivel socioeconómico y de educación lo que justificarían la elevada prevalencia de parasitosis ${ }^{2}$; afectando en su mayoría a individuos en edades pediátricas posiblemente por su inmadurez inmunológica y la falta de correctos hábitos higiénicos ${ }^{3}$.

Se estima que una de cada tres personas está infectada por geohelmintos y cerca de 46 millones de niños entre 1 y 14 años están en riesgo de infectarse por estos parásitos, aproximadamente 13 millones de niños en edad pre-escolar (1 a 4 años) y 33,3 millones de escolares (de 5 a 14 años). Siendo la infección más frecuente en mujeres y niños ${ }^{4}$.

En un meta-análisis realizado en países sudamericanos que recoge data de manuscritos publicados entre los años 1984 al 2011, se informa que Venezuela tiene prevalencia de Trichuris trichuria de 31,8\% seguido de Ascaris lumbricoides de $29,7 \%$ y Uncinarias de $8,2 \%$. Como conclusión, estas tres parasitosis intestinales han mantenido sus prevalencias a pesar de los avances tecnológicos y médicos ${ }^{5}$.

En la materia fecal se excretan las formas infectantes de los parásitos intestinales que pueden luego ser ingeridas directa $o$ indirectamente. La principal forma de transmisión de protozoarios patógenos como Giardia lamblia y Cryptosporidium spp, es el consumo de agua y de alimentos contaminados. Sin embargo, también puede ocurrir la transmisión de persona a persona o de animales a seres humanos ${ }^{6}$.

Actualmente existen tres grupos de parásitos intestinales, que afectan con mayor frecuencia al hombre; los chromistas ${ }^{7}$ y los protozoarios de transmisión hídrica y los nemátodos transmitidos por el suelo.

En Venezuela las enfermedades infecciosas y parasitarias ocupan un lugar importante en la mortalidad postnatal, y dentro de mortalidad total prevalece con 3,93\% para el año $2013^{9}$.

Contribuyen al aumento de casos de malnutrición en niños, lo que conduce al retraso del crecimiento y desarrollo, así como también a la disminución de la capacidad cognitiva ${ }^{10}$.

Generalmente se observan síntomas de intensidad y duración variable, dentro de ellos resulta más frecuentes dolor abdominal de localización inespecífica, diarrea, distensión abdominal, hiperorexia, náuseas y flatulencia, de igual manera la persona puede referir malestar general, prurito anal, picor nasal, insomnio, fatiga y debilidad, puede haber fiebre, y pérdida de peso ${ }^{11}$.

Los niños que reciben tratamiento muestran un considerable incremento de la memoria, de comprensión a lecturas y razonamiento ${ }^{10}$.

Por lo antes referido, en esta investigación se plantea como objetivo; determinar la prevalencia de parasitosis intestinales en niños menores de 15 años en la comunidad rural de Apostadero de Barrancas, del Orinoco; esperando así, contribuir al estudio de parásitos intestinales del estado Monagas, Venezuela.

\section{MATERIALES Y MÉTODOS}

Tipo de estudio: observacional, descriptivo y de corte transversal. Se llevó a cabo en Apostadero una comunidad rural- fluvial ubicada a orillas del Río Orinoco, a 15 minutos al este de Barrancas del Orinoco, municipio Sotillo. Estado Monagas, Venezuela, durante Julio 2015. Esta población está compuesta por criollos, donde las condiciones socio-sanitarias son precarias, las disposiciones de excretas se realizan en pozos sépticos y otros al aire libre, la fuente de agua para consumo se obtiene mediante pozo perforado y es almacenada en un tanque de agua elevado aproximadamente 20 metros de altura desde donde parte el suministro de agua hacia las viviendas por medio de tuberías, las calles son de tierra y no cuentan con un adecuado vertedero de basura.

Universo y muestra: el universo estuvo representado por 120 personas menores de 15 años, según el censo realizado por el Concejo Comunal de la localidad en Junio 2015. La muestra no probabilística, estuvo conformada por 64 niños cuyos representantes firmaron el consentimiento informado; y que quisieron de manera voluntaria participar en el estudio, cumpliendo con el criterio de inclusión, ser menores de 15 años. Las variables del estudio fueron: la presencia de endoparásitos, las especies parasitarias y sus asociaciones, y las manifestaciones clínicos presentes al momento de la evaluación.

Método de recolección de datos: cada participante fue evaluado clínicamente y mediante un análisis coproparasitologico. A través de interrogatorio dirigido a los representantes y examen físico de cada participante se obtuvieron 
las manifestaciones clínicas, y para el estudio coproparasitológico se recolectó en envases apropiados, una muestra de heces obtenida por evacuación espontánea. El análisis de las heces se realizó mediante un examen directo en solución salina fisiológica y coloración temporal con lugol, la aplicación de la Técnica de Kato y la coloración de Kinyoun. La Organización Mundial de la Salud (OMS) recomienda la técnica de KatoKatz para el diagnóstico cualitativo y cuantitativo de las infecciones intestinales humanas por geohelmintos ${ }^{12}$.

Análisis estadístico: los datos fueron analizados por medio del programa estadístico SPSS versión 19 para Windows y Microsoft Excel 2010. Se aplicaron estadísticas descriptivas de frecuencias y porcentajes con tablas de una y doble entradas según sea el caso, se aplicó la prueba de chi cuadrado para mostrar las variables cuya asociación sea significativa con un valor de $\mathrm{p}<0,05$.

\section{RESULTADOS}

Se evaluaron 64 niños entre 0-15 años de edad, de ellos; $51,6 \%(n=33)$ eran masculinos y $48,4 \%$ $(n=31)$ femeninos. La prevalencia global de parasitosis intestinales fue 92,20\% $(n=59 / 64)$. No se encontró diferencia significativa por grupo etario. Se diagnosticaron 14 especies parasitarias de ellos, 1 era chromista y protozoario y 5 eran helmintos. Aunque se aplicó la coloración de Kinyoun para coccidios intestinales, no se encontró especies parasitarias. Los enteroparásitos más frecuentes fueron Ascaris lumbricoides con 72,9\% $(\mathrm{n}=43 / 59)$, seguido de Blastocystis spp. 50,8\%(n=30/59) y Trichuris trichiura $47,5 \%(\mathrm{n}=28 / 59)$ (ver tabla 1$)$.

De los infectados, $18,6 \%(\mathrm{n}=11 / 59)$ resultaron monoparasitados mientras que $81,4 \%(n=48 / 59)$ estaban poliparasitados siendo el género femenino más afectado $96,3 \%(\mathrm{n}=26 / 59)$ que el masculino $68,8 \% \quad(n=22 / 59) \quad$ encontrándose diferencias estadísticamente significativas $(\mathrm{p}=0,007)$ (ver tabla 2). De los poliparasitados la asociación más frecuente fue Ascaris lumbricoides + Blastocystis spp. Con 12,5\% (n=6/48) seguido de Entamoeba coli + Ascaris lumbricoides + Tichuris trichiura $6,3 \%(\mathrm{n}=3 / 48)$ y Ascaris lumbricoides + Giardia lamblia $6,3 \% \quad(\mathrm{n}=3 / 48)$. Es de resaltar que Ascaris lumbricoides fue el parásito asociado más frecuente y es el que predomina en esta serie de casos. (ver tabla 3).

Se presentaron manifestaciones clínicas en un $87,5 \%(\mathrm{n}=56)$, siendo el bruxismo el síntoma más frecuente con $69,6 \%(n=39)$ seguido de dolor abdominal con $62,5 \%(n=35)$ (ver tabla 4$)$.

TABLA 1. Prevalencia de Parásitos Intestinales en niños menores de 15 años Apostadero. Municipio Sotillo; Estado Monagas, Venezuela. Julio 2015.

\begin{tabular}{lll}
\hline Chromistas Protozoarios & Frecuencia & Porcentaje \\
\hline Blastocystis spp. & 30 & 50,8 \\
\hline Entamoeba coli & 17 & 28,8 \\
\hline Giardia lamblia & 14 & 23,7 \\
\hline Endolimax nana & 4 & 6,8 \\
\hline Tricomonas hominis & 4 & 6,8 \\
\hline Chilomastix mesnilii & 2 & 3,4 \\
\hline Complejo Entamoeba & 1 & 1,7 \\
\hline lodamoeba butschlii & 1 & 1,7 \\
\hline Balantidium coli & 1 & 1,7 \\
\hline Helmintos & 43 & \\
\hline Ascaris lumbricoides & 28 & 72,9 \\
\hline Trichuris trichiura & 20 & 47,5 \\
\hline Ancilostomideos & 7 & 33,9 \\
\hline Strongyloides stercoralis & 1 & 11,9 \\
\hline Enterobius vermicularis & 59 & 1,7 \\
\hline Total & & 100,0 \\
\hline \hline
\end{tabular}

Prevalencia de Parasitosis Intestinales 
TABLA 2. Tipo de parasitismo según género en niños menores de 15 años, parasitados, de Apostadero; Municipio Sotillo, Estado Monagas, Venezuela. Julio 2015.

\begin{tabular}{|c|c|c|c|c|c|c|}
\hline \multirow{3}{*}{ Parasitismo } & \multicolumn{4}{|c|}{ Género } & \multirow{2}{*}{\multicolumn{2}{|c|}{ Total }} \\
\hline & \multicolumn{2}{|c|}{ Femenino } & \multicolumn{2}{|l|}{ Masculino } & & \\
\hline & $\mathrm{N}$ & $\%$ & $\mathrm{~N}$ & $\%$ & $\mathbf{N}$ & $\%$ \\
\hline Monoparasitados & 1 & 3,7 & 10 & 31,3 & 11 & 18,6 \\
\hline Poliparasitados & 26 & 96,3 & 22 & 68,8 & 48 & 81,4 \\
\hline Total & 27 & 100,0 & 32 & 100,0 & 59 & 100,0 \\
\hline
\end{tabular}

TABLA 3. Asociaciones parasitarias en niños menores de 15 años, poliparasitados, de Apostadero; Municipio Sotillo, Estado Monagas,Venezuela. Julio 2015.

\begin{tabular}{lll}
\hline Asociaciones parasitarias & Frecuencia & Porcentaje \\
\hline Ascaris lumbricoides+ Blastocystis spp. & 6 & 12,5 \\
\hline Entamoeba coli + Ascaris lumbricoides + Tichuris trichiura & 3 & 6,3 \\
\hline Ascaris lumbricoides + Ancilostomideos & 2 & 4,2 \\
\hline Ascaris lumbricoides + Giardia lamblia & 3 & 6,3 \\
\hline Ascaris lumbricoides + Tichuris trichiura + Ancilostomideos & 2 & 4,2 \\
\hline Ascaris lumbricoides + Entamoeba coli + Trichuris trichiura + Ancilostomideos & 2 & 4,2 \\
+ Strongyloides stercoralis & & \\
\hline Ascaris lumbricoides + Ancilostomideos + Trichuris trichiura + Blastocystis & 2 & 4,2 \\
spp & 2 & 4,2 \\
\hline Entamoeba coli + Blastocystis spp & 26 & 54,2 \\
\hline Otras asociaciones* & 26 \\
\hline Total & 48 & $\mathbf{1 0 0 , 0}$
\end{tabular}

*Se encontraron 26 casos con diferentes asociaciones parasitarias.

TABLA 4. Manifestaciones clínicas en niños menores de 15 años de Apostadero; Municipio

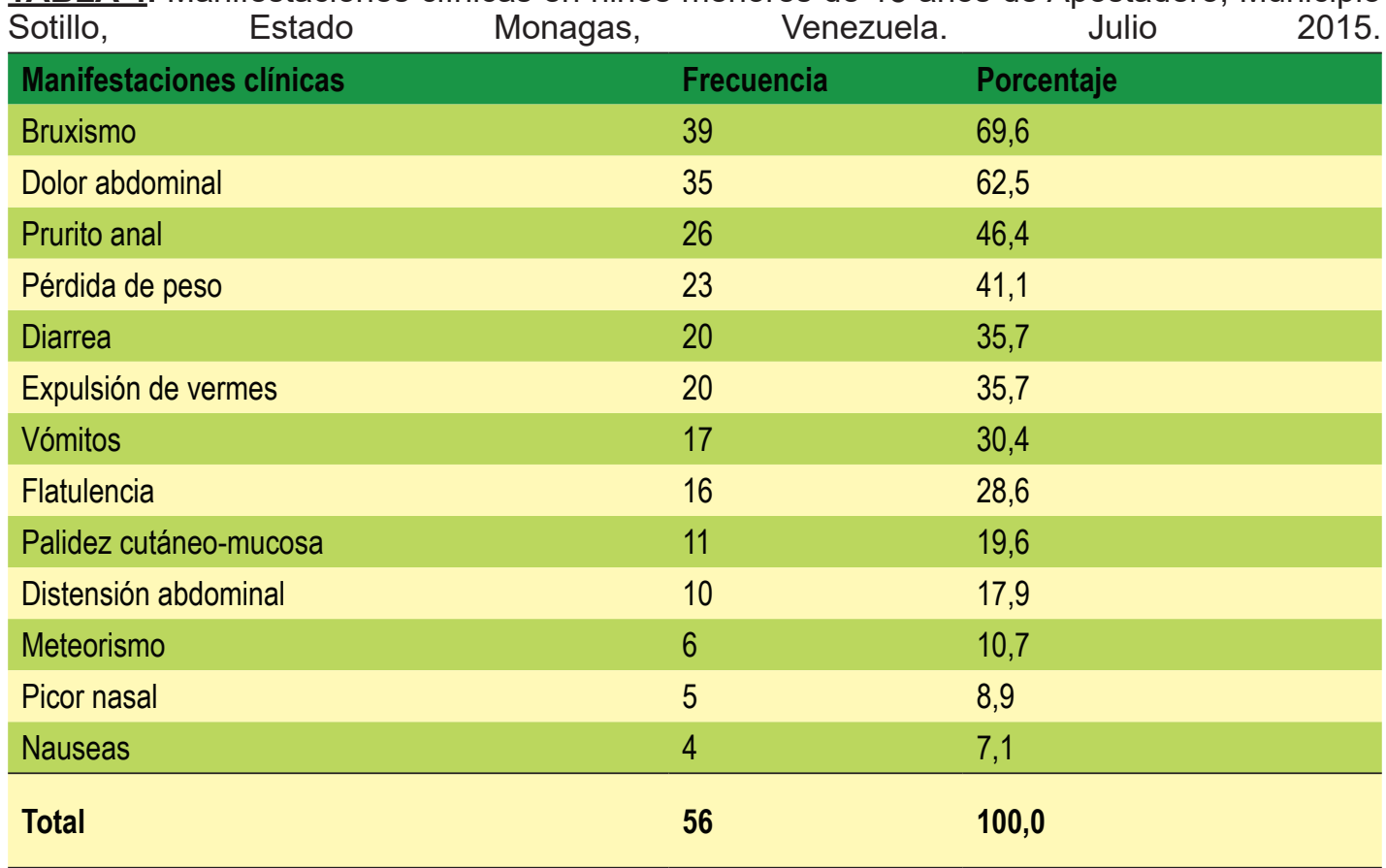




\section{DISCUSIÓN}

Esta investigación demostró parasitosis intestinales en el $92,20 \%$ de la muestra, sin encontrarse diferencias significativas por grupo etario y donde ambos géneros fueron afectados por igual; hallazgos comparables con los obtenidos en el estado Monagas de $91,10 \%$ por Rivas et al. ${ }^{13}$, en Zulia de $86,58 \%$ por Calchi et al. ${ }^{14}$, y en Bolívar $84,3 \%$ por Devera et al. ${ }^{15}$. Del mismo modo, se han señalados resultados menores en diferentes regiones del país, tal es el caso, de Urumaco estado Falcón $78,08 \%$ por Cazorla et al. ${ }^{16}$, en Miranda $64,3 \%$ por Arencibia et al. ${ }^{17}$, en Anzoátegui $60,1 \%$ por Devera et al. ${ }^{18} \mathrm{y}$ en Lara $42,5 \%$ por Fuentes et al. ${ }^{19}$.

Estos valores de alarma se puede atribuir al contexto en el cual viven los habitantes de este tipo de comunidad, tales como: precarias condiciones higiénicas, bajo nivel educativo, carecer de servicios de aguas blancas por tubería y red de cloacas, poco acceso a programas de salud, disposición inadecuada de los desechos sólidos así como problemas en el abastecimiento de agua potable. En resumen, baja educación sanitaria, lo que se traduce en subdesarrollo y pobreza de la población ${ }^{16-20}$.

Las manifestaciones clínicas en el parasitismo intestinal son imprecisas, en este estudio fueron variadas, siendo el bruxismo el predominante en un $69,6 \%$ de los casos de niños con endoparasitosis, que 56 de ellos manifiestan síntomas, resultado comparable con el estudio realizado por Baldeón et al. ${ }^{21}$, en una población de Perú donde se obtuvo prevalencia de 57,1\% en un grupo similar, muy superior al obtenido por Requena et al. ${ }^{22}$, en una población preescolar de Ciudad Bolívar- Venezuela. Seguido al bruxismo, se encontró el dolor abdominal $(62,5 \%)$ y prurito anal $(46,4 \%)$, este último, es síntoma característico de la enterobiosis cuyo agente causal es E. vermicularis, aunque para poder detectarlo es necesaria la prueba de Graham $^{23}$, la cual no fue realizada en este estudio, sin embargo, la abstención de este método no descarta la presencia de dicha parasitosis en la muestra estudiada.

A. Lumbricoides fue el helminto más prevalente con un $72,9 \%$, valor superior al $40,9 \%$ conseguido por Rivas et al. ${ }^{13}$, en un estudio realizado en diferentes zonas del mismo estado, así como con los obtenidos en otras entidades del país por Calchi et al. ${ }^{14}$, y Arencibia et al. ${ }^{17}$ con frecuencia de 44,91\% y 10,3\% respectivamente.

Esta prevalencia puede atribuirse a la estrecha relación de este grupo etario con suelos contaminados y a la ignorancia de las medidas mínimas de higiene que deben ser implementadas para evitar la diseminación de las enteroparasitosis ${ }^{19}$. El segundo más frecuente fue Blastocystis spp con 50,8\%; resultado por debajo a los encontrados por Devera et al. 24 en 2006 de 60\%, y Devera et al. ${ }^{15}$ en 2014 de 65,2\%, en comunidades rurales, al igual que el $64,70 \%$ obtenido por Calchi et al. ${ }^{14}$. Es de resaltar que la taxonomía y sistematización de este parasito un está en discusión, hay autores que lo describen como protozoario y otros como cromista ${ }^{7}$. En tercer lugar por frecuencia, fue hallado $\mathrm{T}$. trichiura con $47,5 \%$, similar resultado obtenidos por González et al. en área rural del estado Sucre $^{25}$ e inferior a los reportados por Calchi et al. $56,68 \%$ en el estado $\mathrm{Zulia}^{14}$ y valor superior a $36,5 \%$ obtenidos por Gil et al. ${ }^{26}$ en el estado Trujillo. En este estudio los helmintos tuvieron una prevalencia superior a los protozoarios $\mathrm{y}$ cromistas, datos que difieren a los encontrados por Calchi et al. ${ }^{14}$. Otro hallazgo importante fue la presencia de los parásitos comensales $E$. coli 28,8\%, E. nana 6,8\% e I. butschlii 1,7\%, resultados que difieren a los resultados obtenidos por otros autores realizados en el país ${ }^{18,24,27}$. En el ámbito clínico éstos protozoos comensales son de ínfimo interés, sin embargo, desde la perspectiva epidemiológica, representa un indicador importante de contaminación fecal en aguas servidas a la población ${ }^{15,18,24}$ De igual forma se encontró la presencia de 1 caso $(1,7 \%)$ de parasitismo por el Complejo Entamoeba compuesto por las especies: histolytica, dispar y moshkovskii. De éstas 3 sólo la primera tiene interés clínico por ser patógena, pero todas por ser morfológicamente iguales son incluidas en el mismo complejo cuando no se cuenta con las herramientas de biología molecular que permitan la diferenciación entre dichas especies ${ }^{29}$. Este resultado es superior a lo descrito por Devera et al. ${ }^{18}$, Otro hallazgo importante fue la presencia de los parásitos comensales E. coli $28,8 \%$, E. nana $6,8 \%$ e $I$. butschlii $1,7 \%$, resultados que difieren a los resultados obtenidos por otros autores realizados en el país; 4,5\% en Bolívar por Devera et al. $^{24}$, y $9 \%$ en Trujillo por Gil et $\mathrm{al}^{26}$.

Es de resaltar la alta frecuencia de individuos poliparasitados $(81,4 \%)$ sobrelosmonoparasitados $(18,6 \%)$ resultados similares a los obtenidos en Bolívar por Devera et al. ${ }^{24}$, aunque difieren con los descritos, en Zulia por Calchi et al. ${ }^{14}$, y en Valencia por Solano et al..$^{30}$, en estos estudios, dichos hallazgos se atribuyen a las condiciones sanitarias antes descritas. De los poliparasitados, 
el género femenino fue afectado en un $96,3 \%$ y el masculino en un $68,8 \%$, siendo esta diferencia estadísticamente significativa, sin embargo, la O.M.S señala al respecto que la prevalencia parasitaria en un determinado género se debe a factores ocupacionales y de comportamiento y no a una susceptibilidad diferencial ${ }^{28}$, otros estudios señalan que no existen factores preponderantes que puedan influir en el padecimiento de infección por parásitos de acuerdo a un determinado género $^{17,18}$; mientras que, factores como: baja condición socio-sanitaria, bajo nivel educativo y pobreza, influyen directamente en la prevalencia de parasitosis intestinal independientemente del género $^{27,31}$.

Del mismo modo, se encontró que la asociación parasitaria más frecuente fue $A$. lumbricoides + Blastocystis spp con $12,5 \%$, seguido por $E$. coli $+A$. lumbricoides $+T$. trichiura y $A$. lumbricoides $+G$. lamblia ambos con el mismo porcentaje de prevalencia $6,3 \%$. Es de resaltar que A. lumbricoides fue el parásito asociado más frecuente y en las otras 26 asociaciones y es el que predomina en esta serie de casos. La alta frecuencia de helmintos, cromistas, protozoarios y la combinación de estos, nos permiten deducir que el agua es el principal agente de transmisión, ya que los huevos embrionados de $A$. lumbricoides contaminan suelos y alimentos por medio del agua $^{20,23}$, y en el caso de los protozoos se sabe que cuentan con características importantes que le dan la habilidad de convertirse en agentes etiológicos de enfermedades transmisibles por esta vía, poseen estabilidad al medio ambiente, infectan aún con carga parasitaria mínima, y crean resistencia a los agentes químicos usados para la potabilización del agua ${ }^{15,18,19,32}$.

Una vez finalizado el estudio cada individuo recibió por escrito sus resultados coproparasitológicos, aquellos que resultaron parasitados se les suministró drogas antiparasitarias específicas para su tratamiento, de igual forma, fueron dictadas charlas educativas a los distintos miembros de la comunidad. Todavía el parasitismo juega un papel importante en la morbilidad de los pobladores de los sectores rurales del país, la solución a este problema está dirigida a mejorar condiciones socio económicas y sanitarias, aplicar medidas higiénicas (lavado de manos y alimentos, hervir el agua de consumo) y sobre todo modificar hábitos y costumbres; sin embargo su ejecución es difícil, es por ello que es necesario realizar proyectos de mediano y largo plazo en conjunto con los entes gubernamentales y no gubernamentales, sector privado y comunidad en general para que estas medidas sean aplicadas correctamente y así reducir la incidencia de esta patología y mejorar la calidad de vida de sus habitantes.

\section{CONCLUSIONES}

Se determinó una alta prevalencia de parasitosis intestinales $(92,20 \%)$ en niños menores de 15 años de Apostadero, estado Monagas, siendo el bruxismo la manifestación clínica más prevalente con $69,6 \%$, seguido de dolor abdominal con 62,5 $\%$ y prurito anal con 46,4\%. Los helmintos más frecuentes fueron: A. lumbricoides, T. trichiura y Ancilostomideos con 72,9\%, 47,5\% y 33,9\% respectivamente.

Entre los cromistas y protozoarios destacaron: Blastocystis spp (50,8\%), E. coli $(28,8 \%)$ y $G$. lamblia $(23,7 \%)$. El poliparasitismo fue superior con $81,4 \%$ al monoparasitismo con $18,6 \%$ y las asociaciones más frecuentes fueron: $A$. lumbricoides + Blastocystis spp. con 12,5\%.

\section{AGRADECIMIENTOS}

A la comunidad de Apostadero por la disponibilidad y aceptación del estudio al señor Ernesto Castillo por ser guía dentro de la comunidad, a la Dra. Marelys Rodríguez de Salud Ambiental en Maturín del Estado Monagas por la donación de los medicamentos, al Departamento de Parasitología y Microbiología de la Escuela de Ciencia de la Salud de la Universidad de Oriente Núcleo Bolívar por los equipos de microscopio y reactivos que se utilizaron para procesar las muestras, a la Licenciada Rosario Tutaya, al Licenciado Jose Nastasi Miranda, a los universitarios Javier Cedeño, Yoliber Marcano, Modesto Pimentel y al Comité Permanente de Evaluación y Desarrollo Científico (CPEDEC) de la Sociedad Científica de Estudiante de Medicina de Universidad de Oriente Núcleo Bolívar gestión 2014-2015 por su colaboración en el trabajo de campo.

\section{REFERENCIAS}

1. Chacin L. Las enfermedades parasitarias intestinales como un problema de salud global. Investigación Clínica 2013. 54(1):1-4. Acceso 29 de octubre 2017. Disponible en: https://goo.gl/5Uywsa

2. Nicholls S. Parasitismo intestinal y su relación con el saneamiento ambiental y las condiciones sociales en latinoamérica y el -Caribe. Biomédica Colombia. 2016; 36(4). Acceso 29 de octubre 2017. Disponible en: https://goo.gl/UyDMC8

3. Barón M, Solano L, Paez M, Pabon M. Estado nutricional de hierro y parasitosis intestinal en niños de Valencia, Estado Carabobo, Venezuela. An Venez de Nutr:2007; 20 (1):5-11. Acceso 29 de octubre 2017. Disponible en: https://goo.gl/7fmiDS PAHO/WHO. Geohelmintiasis. Acceso 20 de septiembre 2017. Disponible en: http://www.paho.org/hq/index. 
php?option=com_topics\&view=article\&id=419\&/temid=41001\&lang=es

4. Chammartin F, Scholte R, Guimarães L, Tanner M, Utzinger J, VounatsouP. Soil-transmitted helminth infection in South America: a systematic review and geostatistical meta-analysis. Lancet Infect Dis. 2013; 13:50718. Acceso 29 de octubre 2017. Disponible en: http://www.thelancet.com/ journals/laninf/article/PIIS1473-3099(13)70071-9/fulltext

5. Speich B, Croll D, Fürst T, Utzinger J, Keiser J. Effect of sanitation and water treatment on intestinal protozoa infection: a systematic review and meta-analysis. Lancet Infect Dis. 2016;16(1):87-99. Acceso 29 de octubre 2017. Disponible en: http://www.thelancet.com/journals/laninf/article/ PIIS1473-3099(15)00349-7/fulltext

6. Devera R. ¿Blastocystis spp. O B. hominis? ¿PROTOZOARIO O CHROMISTA?. Saber Universidad de Oriente, Venezuela. 2014; 26(4):48890. acceso 29 de octubre 2017. Disponible en: http://www.scielo.org.ve/pdf/ saber/v26n4/art14.pdf

7. Nastasi-Miranda J, Blanco Y, Aray R, Rumbos E, Vidal-Pino M, Volcán I. Ascaris lumbricoides $Y$ Otros Enteroparásitos En Niños De Una Comunidad Indígena Del Estado Bolívar, Venezuela. CIMEL. 2017; 22(1):40-5. Acceso 29 de octubre 2017. Disponible en: https://www.cimel. felsocem.net/index.php/CIMEL/article/view/741/388

8. Ministerio de Poder Popular Para la Salud. Anuario de mortalidad 2013. Caracas. Venezuela. 2015. Acceso 17 julio 2017. Disponible en: http:// www.ovsalud.org/descargas/publicaciones/documentos-oficiales/AnuarioMortalidad-2013.pdf

9. OMS. Alerta sobre infección de parásitos intestinales en países en desarrollo. 2008. Centro de noticias ONU. Acceso 17 septiembre 2017. Disponible en: $h$ ttp://www.un.org/spanish/News/story.asp?News/D=13222\#

10. Cardona-Arias JA, Rivera-Palomino Y, Carmona-Fonseca J. Salud indígena en el siglo XXI: parásitos intestinales, desnutrición, anemia y condiciones de vida en niños del resguardo indígena CañamomoLomaprieta, Caldas-Colombia. MÉD. UIS. 2014;27(2):29-39. Acceso 29 de octubre 2017. Disponible en: http://revistas.uis.edu.co/index.php/ revistamedicasuis/article/view/4322/4602

11. World Health Organization. Laboratory diagnosis of helminth parasites. Training manual on diagnosis of intestinal parasites: tutor's Guide. Geneva: 2004. Acceso 29 de octubre 2017. Disponible en: http://apps.who. int/iris/bitstream/10665/69987/1/WHO_CTD_SIP_98.2.pdf

12. Rivas J, Ramos R, Rodríguez M, Espinoza J. Prevalencia de Ascaris lumbricoides y otros enteroparásitos en habitantes de 12 comunidades rurales del estado Monagas- Venezuela. Médico de Familia. 2005; 13(2): 23-31. Acceso 10 agosto 2016. Disponible en: http://www.sovemefa.com/ revistas_sovemefa/medico_de_familia_13_2_2005.pdf

13. Calchi M, Rivero Z, Acurero E, Díaz I, Chourio G, Bracho A, et al. Prevalencia de enteroparasitos en dos comunidades de Santa Rosa de Agua en Maracaibo, Estado Zulia- Venezuela 2006. Kasmera. 2007; 35(1):38-48. Acceso 29 de octubre 2017. Disponible en: http:// produccioncientificaluz.org/index.php/kasmera/article/view/4783/4777

14. Devera R, Blanco Y, Amaya I, Álvarez E, Rojas J, Tutaya $R$, et al. Prevalencia de parásitos intestinales en habitantes de una comunidad rural del estado Bolívar- Venezuela. Kasmera. 2014; 42(1):22-31. Acceso 29 de octubre 2017. Disponible en: http://produccioncientificaluz.org/index. php/kasmera/article/view/8651/8641

15. Cazorla D, Leal G, Escalona A, Hernández J, Acosta M, Morales $P$. Aspectos clínicos y epidemiológicos de la infección por coccidios intestinales en Uramaco, estado Falcón. Bol. Malariol. Salud ambiental. 2014; 54 (2):159-73. Acceso 29 de octubre 2017. Disponible en: http://www. scielo.org.ve/pdf/bmsa/v54n2/art06.pdf

16. Arencibia H, Lobaina J, Terán C, Legra R, Arencibia A. Parasitismo intestinal en una población infantil venezolana. Medisan. 2013; 17 (5): 742-48. Acceso 29 de octubre 2017. Disponible en: http://www.redalyc.org/ articulo.oa?id=368444992001

17. Devera R, Blanco Y, Amaya I, Nastasi M, Rojas G, Vargas B. Parásitos intestinales en habitantes de la comunidad rural "La Canoa" Estado Anzoátegui Venezuela. Revista Venezolana de Salud Pública. 2014; 2 (1): 15-21. Acceso 29 de octubre 2017. Disponible en: http://bibvirtual.ucla.edu. ve/db/psm_ucla/edocs/rvsp/RVSP0201/articulo2.pdf

18. Fuentes M, Galindez L, García D, Gonzalez N, Goyanes J, Herrera E, et al. Frecuencia de parasitosis y características epidemiológicas de la población infantil de 1 a 12 años que consultan el ambulatorio Urbano tipo II de cerro Gordo. Barquisimeto, estado Lara. Enero-Junio 2007. Kasmera. 2011; 39 (1): 31-42. Acceso 29 de octubre 2017. Disponible en: http://produccioncientificaluz.org/index.php/kasmera/article/view/4872/4864

19. Botero J, Zuluaga N. Nemátodos intestinales de importancia médica en Colombia. latreia. 2001, 14 (1): 47-56. Acceso 29 de octubre 2017. Disponible en: https://aprendeenlinea.udea.edu.co/revistas/index.php/iatreia/ article/view/3792/3508

20. Baldeón C, Del Castillo C, Balarezo J, Alva A, Sánchez P, Velázquez J. Bruxismo y Parasitosis intestinal en niños de 4 a 6 años de edad en La Brea (Talara, Piura) Perú. Rev Estomatol Herediana. 2014; 24 (3): 163-70. Acceso 29 de octubre 2017. Disponible en: http://www.upch.edu.pe/vrinve/ dugic/revistas/index.php/REH/article/view/2091/2081

21. Requena I, Lizardi V, Mejía L, Castillo H, Devera R. Infección por enterobiusvermicularis en niños preescolares de Ciudad Bolívar, Venezuela. Rev Biomed. 2002; 13(4):231-40. Acceso 29 de octubre 2017. Disponible en: http://www.revistabiomedica.mx/index.php/revbiomed/article/ view/322/334

22. Botero D, Restrepo M. Parasitosis intestinales por nematodos. Botero D, Restrepo M. Parasitosis Humanas. $5^{a}$ Edición. Medellin, Colombia. Editorial Corporación para Investigaciones Biológicas; 2012.p177-86.

23. Devera R, Angulo V, Amaro E, Finali M, Franceschi G, Blanco Y, et al. Parásitos intestinales en habitantes de una comunidad rural del estado Bolívar, Venezuela. Rev Biomed. 2006;17(4):259-68. Acceso 29 de octubre 2017. Disponible en: http://www.revistabiomedica.mx/index.php/revbiomed/ article/view/465/476

24. González B, Michelli E, Guilarte D, Rodulfo H, Mora L, Gómez T. Estudio comparativo de parasitosis intestinales entre poblaciones rurales $y$ urbanas del estado Sucre, Venezuela. Rev. Soc. Ven. Microbiol. 2014; 34(2):97-102. Acceso 29 de octubre 2017. Disponible en: http://saber.ucv.ve/ ojs/index.php/rev_vm/article/view/8002/7909

25. Gil M, Pineda G, Alvis M, Durán E, Guédez C, Castillo C. Helmintiasis Intestinales en escolares de varias instituciones públicas de la parroquia la Paz del municipio Pampán-Trujillo, Venezuela. Revista Academia. 2013; 12 (25):5-10. Acceso 29 de octubre 2017. Disponible en: http://www.saber.ula. ve/bitstream/123456789/38406/1/articulo1.pdf

26. Marcano Y, Suarez B, González M, Gallego L, Hernandez T, Naranjo M. Caracterización epidemiológica de parasitosis intestinales en la comunidad 18 de mayo, Santa Rita, estado Aragua- Venezuela, 2012. Bol. Malariol. salud ambiental. 2013; 53 (2): 135-45. Acceso 29 de octubre 2017. Disponible en: $h$ ttp://www.scielo.org.ve/pdf/bmsa/v53n2/art03.pdf

27. Organización Mundial de la Salud. Infecciones intestinales por protozoos y helmintos. Edit. Gráficas Reunidas, Serie informes técnicos 666. 1981;7-66. Disponible en: http://apps.who.int/iris/bitstream/10665/41310/1/ WHO_TRS_666_\%28part1\%29_spa.pdf

28. Chacín L. Amebiasis: aspectos clínicos, terapéuticos y de diagnóstico de la infección. RevMedChil. 2013; 141(5):609-15. Acceso 29 de octubre 2017. Disponible en: http://www.revistamedicadechile.cl/ojs/index. php/rmedica/article/view/2078/165

29. Solano L, Acuña I, Barón M, Morón A, Sánchez A. Asociación entre pobreza e infestación parasitaria intestinal en preescolares, escolares y adolescentes del sur de Valencia, estado Carabobo-Venezuela. Kasmera. 
2008; 36(2):137-47. Acceso 29 de octubre 2017. Disponible en: http:// produccioncientificaluz.org/index.php/kasmera/article/view/4831/4824

30. Del Valle E, El Hen F, Garantón A, Marin L. Aspectos epidemiológicos y hematológicos asociados a las parasitosis intestinales en indígenas warao de una comunidad del estado Sucre-Venezuela. Interciencia. 2014; 39 (2): 116-21. Acceso 29 de octubre 2017. Disponible en:. http://www.redalyc. org/html/339/33930068007/
31. Lacoste E, Rosado F, Ángel C, Rodríguez M, Medina I, Suárez R. Aspectos epidemiológicos de las parasitosis intestinales en niños de Vegón de Nutrias, Venezuela. Revista Cubana de Higiene y Epidemiología. 2012; 50 (3): 330-39. Acceso 29 de octubre 2017. Disponible en: http://www. redalyc.org/pdf/2232/223225493004.pdf 